J. Clin. Chem. Clin. Biochem.

Vol. 15, 1977, pp. 657-662

\title{
Quantitative gaschromatographische Bestimmung von aromatischen Carbonsäuren mit Hilfe von Glas-Kapillarsäulen
}

\author{
Von $K$. Olek und P. Wardenbach ${ }^{1}$ ) \\ Institut für Humangenetik, Universität Bonn
}

(Eingegangen am 25. November 1976/26. Mai 1977)

Zusammenfassung: Am Beispiel von 20, möglicherweise im Urin vorkommender aromatischer Carbonsäuren wird gezeigt, daß die Trennung mit einer Glas-Kapillarsäule, verglichen mit einer gepackten Säule, eine wesentlich höhere Trennleistung bei verkürzter Analysenzeit bietet. Trotz der Verwendung eines Splitsystems sind Reproduzierbarkeit und Linearität der quantitativen Bestimmung mit der mit gepackten Säule vergleichbar. Es wird eine größere Nachweisempfindlichkeit erreicht. Die Vorteile bei der Anwendung auf die Probleme der Diagnostik angeborener Stoffwechselanomalien werden an Urinanalysen von Phenylketonurie-Patienten gezeigt.

\section{Quantitative determination of aromatic carboxylic acids with glass-capillary-columns}

Summary: With a glass-capillary column 20 aromatic acids, probably present in urine, were analysed quantitatively. In comparison with a packed column the capillary column offers several advantages: a higher resolution; a greatly reduced analysis time, an increased sensitivity. Though a split system in used, repeatability and linearity are suitable for quantitative analysis. The advantages are best recognized by the analysis of urine specimens of patients with a metabolic disorder (phenylketonuria).

\section{Einleitung}

In den letzten Jahren gewinnt die Diagnose angeborener Stoffwechselerkrankungen aufgrund von Urinuntersuchungen zunehmend an Bedeutung. Wegen ihrer verhältnismäßig hohen Frequenz besitzen die Störungen im Stoffwechsel der aromatischen Aminosäuren eine herausragende Stellung. Beim Vorliegen von Homozygotie ist eine quantitative Bestimmung der durch den Defekt betroffenen Metaboliten mit gepackten Säulen meist problemlos. Daß eine gepackte Säule nicht in der Lage ist, alle vorhandenen Substanzen vollständig żu trennen, wird erst dann bedeutsam, wenn man geringfügige metabolische Abweichungen untersuchen will, wie etwa die Heterozygotie rezessiver Anomalien oder variante Formen der Hyperphenylalaninämie. Als Ausw̌eg bietet sich die Kombination verschiedener chromatographi: scher Prinzipien, die Anwendung hochspezifischer Detektionssyssteme oder die Verwiendung eines hochauflösenden Trennsystems an, wie es die Glas-Kapillare darstellt. Im Folgenden wird die qualitative und quantitative Analyse einiger Harnmetaboliten mit einer GlasKapillare beschrieben.

1). Diese Arbeit wurde von der Deutschen Forschungsgemein= schaft unterstützt.

\begin{abstract}
Methodik
Aufstellen der Standardkurven

Jeweils $10 \mathrm{mg}$ der Standardsubstanzen werden abgewogen und in $100 \mathrm{ml}$ Methanol gelöst. Nach dem Abpipettieren von 1, 3, $5,10,15,20$ und $25 \mathrm{ml}$ werden diese Lösungen am Rotationsverdampfer unterhalb $40^{\circ} \mathrm{C}$ bis zur Trockene eingedampft, in $1 \mathrm{ml}$ Reaktionslösung, bestehend aus N, O-Bis-trimethylsilylacetamid, Chloroform und Acetonitril im Volumenverhältnis $1+1+2$, aufgenommen und 1 Stunde zur Vervollständigung der Silylierung auf $40^{\circ} \mathrm{C}$ erwärmt (Reacti-Therm, Pierce). Die Silylierungslösung enthält $1 \mu \mathrm{l} / \mathrm{ml} n$-Tetradecan als inneren Standard. Jeweils $0,5 \mu l$ der erhaltenen Lösung werden injiziert. Dieser Vorgang wird zur Ausschaltung zufalliger Fehler $5 \mathrm{mal}$ wiederholt. Die vom Integrator ausgedruckten Impulszahlen werden durch die Impulszahlen des inneren Standards dividiert, um den Einspritzfehler auszuschalten. Die so erhaltenen relativen Impülszahlen werden mit Hilfe des Nalimow-Tests (1) von Ausreißern befreit. Die Standardgerade wird durch eine lineare Regressionsanalyse (2) ermittelt, wobei die Zielgröße oder abhängige Variable die relative Impulszahl darstellt und die Einflußgröße (unabhängige Variable) durch die in $1 \mathrm{ml}$ Reaktionslösung enthaltene Substanzmenge gegeben ist.
\end{abstract}

Extraktion von Urinproben

$20 \mathrm{ml}$ Urin werden mit $5 \mathrm{ml}$ halbkonzentrierter Salzsäure versetzt. $20 \mathrm{ml}$ dieser Lösung (entspricht $16 \mathrm{ml}$ Urin) werden auf eine Kieselgel-Fertigsäule (Extrelut; Fa. Merck, Darmstadt) pipettiert. Nach dem Einziehen der wäßrigen Phase werden die lipophilen Substanzen mit $40 \mathrm{ml}$ Essigsäureethylester eluiert. Die Essigsäureethylester-Lösung wird dann am Rotationsverdampfer bis zur Trockene eingedampft und der Rückstand in 
Tab. 1. Extraktionsverlust der Phenylalanin-Metaboliten.

\begin{tabular}{llllll}
\hline Substanz & $\begin{array}{l}\text { Einwaage } \\
(\mathrm{mg})\end{array}$ & $\begin{array}{l}\text { wiedergefunden } \\
(\mathrm{mg})\end{array}$ & $\begin{array}{l}\text { Bereich } \\
(\mathrm{mg})\end{array}$ & $\begin{array}{l}\text { Variations- } \\
\text { koeffizient } \\
(\%)\end{array}$ \\
\hline Phenylessigsäure & 0,82 & $\begin{array}{c}0,92 \\
(112,2 \%)\end{array}$ & $0,81-0,99$ & 5,08 & 9 \\
Mandelsäure & 0,85 & $\begin{array}{c}0,77 \\
(90,6 \%)\end{array}$ & $0,71-0,81$ & 4,09 & 9 \\
o-Hydroxyphenylessigsäure & 0,90 & $\begin{array}{c}0,81 \\
(90,0 \%)\end{array}$ & $0,78-0,84$ & 2,49 & 7 \\
3-Phenylmilchsäure & 0,84 & $\begin{array}{c}0,93 \\
(110,7 \%)\end{array}$ & $0,85-1,01$ & 5,88 & 9 \\
p-Hydroxyphenylessigsäure & 1,06 & $\begin{array}{c}1,04 \\
(98,1 \%)\end{array}$ & $0,92-1,16$ & 7,55 & 7 \\
Phenylbrenztraubensäure & 0,86 & $\begin{array}{c}0,57 \\
(66,3 \%)\end{array}$ & $0,54-0,60$ & 3,81 & 7 \\
\hline
\end{tabular}

$1 \mathrm{ml}$ Silylierungslösung (siehe oben) aufgenommen. Nachdem die Mischung 1 Stunde auf $40^{\circ} \mathrm{C}$ erhitzt worden ist, werden $0,5 \mu l$ der erhaltenen Lösung injiziert.

\section{Bestimmung des Extraktionsverlustes}

$20 \mathrm{ml}$ Urin werden wie oben beschrieben aufbereitet und gaschromatographisch analysiert. Diese Daten ergeben die Leerwerte. Dann wird in $20 \mathrm{ml}$ des gleichen Urins eine definierte Menge Standardsubstanzen gelöst. Nach der Aufarbeitung wird die Probe quantitativ vermessen. Von den Peakflächen (Impulszahlen) der einzelnen Standardsubstanzen werden die entsprechenden Leerwerte abgezogen. Diese Differenz wird mit der zu erwartenden Impulszahl (aus der Standardkurve) verglichen und daraus der Extraktionsverlust berechnet (Tab. 1).

\section{Gaschromatographie}

\section{Chromatographie mit der gepackten Säule}

Gerät: Ein Pye 104 Doppelsäulengerät mit beheiztem Flammenionisationsdetek tor, Einlaßheizung und Unicam S4 Probenaufgabeautomatik; Glassäule $9 \mathrm{ft} \times 2 \mathrm{~mm}$ i. d., gefullt mit $3 \%$ OV-3 auf Chromosorb WHP 100-120 mesh; Temperaturprogramm: Anfangsisotherme $5 \mathrm{~min} 100^{\circ} \mathrm{C}$; Steigerungsrate $4^{\circ} \mathrm{C}$ pro Minute bis $250^{\circ} \mathrm{C}$; Endisotherme $5 \mathrm{~min} 250^{\circ} \mathrm{C}$. Der Detektor wurde auf $300^{\circ} \mathrm{C}$, die Einlaßheizung auf $190^{\circ} \mathrm{C}$ gehalten. Gasströme: Wasserstoff $28 \mathrm{ml} / \mathrm{min}$, Luft $700 \mathrm{ml} / \mathrm{min}$; Stickstoff $12 \mathrm{ml} / \mathrm{min}$. Verstärkung $8 \times 10^{2}$. Retentionszeiten und Impulszahlen lieferte ein elektronischer Integrator (Vidar Mod 6300); Registrierung der Chromatogramme erfolgte mit dem Unicam AR-25 bei einem Papiervorschub von $90 \mathrm{~s} / \mathrm{cm}$.

\section{Chromatographie mit der Glaskapillare}

Es wurde ein Pye Unicam 104 Gaschromatograph mit unbeheiztem Flammenionisationsdetek tor verwendet. Die Trennung erfolgte auf einer $25 \mathrm{~m} \times 0,23 \mathrm{~mm}$ Glaskapillare, belegt mit OV-101. Die Glaskapillare und der vorgeschaltete Allglas-Injektions-Splitter, entgegen den werkseitigen Vorschlägen nicht mit einer Füllung versehen, wurden von LKB-Instruments, Schweden, bezogen. Versuche, die silylierten Carbonsäuren auf Glaskapillaren anderer Hersteller zu vermessen, schlugen bisher fehl. Um einen konstanten Säulenvordruck aufrecht zu erhalten, wurde der serienmäßige Durchflußregler durch einen PräzisionsDruckregler ersetzt (R/182 N, Negretti und Zambra). Die Zuführung von $40 \mathrm{ml}$,"make-up"-Gases am Ende der Trennkapillare mit Hilfe eines T-Stücks steigerte die Detektorenempfindlichkeit um über $100 \%$. Alle in den Detektor einströmenden Gase, Wasserstoff $(43 \mathrm{ml} / \mathrm{min})$, Luft $(700 \mathrm{ml} / \mathrm{min})$ und ,makeup"-Gas $(40 \mathrm{ml} / \mathrm{min})$ wurden zur Erzielung einer stabilen Basislinie mit Hilfe von Molekularsieb (Hydro-Purge-Gas-Filters, Applied Science Laboratories) von organischen Verunreinigungen befreit. Folgende Trennsäulenbedingungen kamen zur Anwendung; Trägergasstro $0,78 \mathrm{ml} / \mathrm{min}$, Stickstoff bei einem Säulenvordruck von 0,8 bar bei $100^{\circ} \mathrm{C}$ (Optimum der Trenn- stufenzahl); Einspritzblocktemperatur $250^{\circ} \mathrm{C}$; Anfangsisotherme $1 \mathrm{~min} 100^{\circ} \mathrm{C}$; Steigerungstate $10^{\circ} \mathrm{C}$ pro Minute bis $250^{\circ} \mathrm{C}$ Endisotherme 5 Minuten $250^{\circ} \mathrm{C}$; Splitverhältnis $1: 46,91$, Verstärkung $1 \times 10^{2}$. Die Chromatogramme wurden bei einem Papiervorschub von $48 \mathrm{~s} / \mathrm{cm}$ aufgenommen; Retentionszeiten und Impulszahlen lieferte ein elektronischer Integrator (Vidar, Modell 6300).

\section{Ergebnisse}

Abbildung 1 und 2 zeigen die Gaschromatogramme von silylierten aromatịschen Carbonsäuren, die mit der gepackten (Abb. 1), bzw, der Gläs-Kapillarsäule (Abb. 2), unter den im experimentellen Teil erwähnten Bedingungen erhalten wurden. Die verbesserte Trennqualität der Glaskapillare zeigt sich daran, daß mit ihr $o$-Hydroxyphenylessigsäure, 3-Phenylmilchsäure und $m$-Hydroxyphenylessigsäure zu $100 \%$ aufgelöst werden, während auf der gepackten Säule nur eine Auflösung von ungefähr $90 \%$ erzielt wird. Weiterhin sind auf der gepackten Säule Vanillinsäure und $o$-Hydroxyhippursäure nur als Schulter von 2,6-Dihydroxybenzoesäure bzw. Ferulasäure zu erkennen; 2,4-Dihydroxybenzoesäure und 0 -Hydroxyzimtsäure erscheinen als ein verbreiteter Peak (Abb. 1). In allen Fällen zeigt die Kapillarsäule eine Auflösung von weit über $90 \%$. Der Gewinn an Trennqualität wird noch besser erkennbar beim Vergleich von Urinchromatogrammen (Abb. 3-6), die auch die gesteigerte Nachweisempfindlichkeit deutlich machen. Ein weiterer Vorteil besteht in der um 15 Minuten verkürzten Analysenzeit.

Zur Demonstration der erzielten Reproduzierbarkeit von relativen Retentionszeiten ( $n$-Tetradecan $=1,00$ ), wesentlich für die gaschromatographische Identifizierung von Substanzen in biologischen Proben, sei eine Substanz mit kurzer (Mandelsäure) und eine mit langer Retentionszeit ( $m$-Hydroxyzimtsäure) herausgegriffen:

$\mathrm{R}_{\mathrm{t}}$ (Mandèlsäure):

$\bar{x}_{0}=1,110^{\circ} \mathrm{s}=0,002 \quad \mathrm{n}=20$

$\overline{\mathrm{R}}_{\mathrm{t}}$ (m-Hydroxyzimtsäure):

$\bar{x}=1,689 \quad s=0,006 \quad n=20$. 


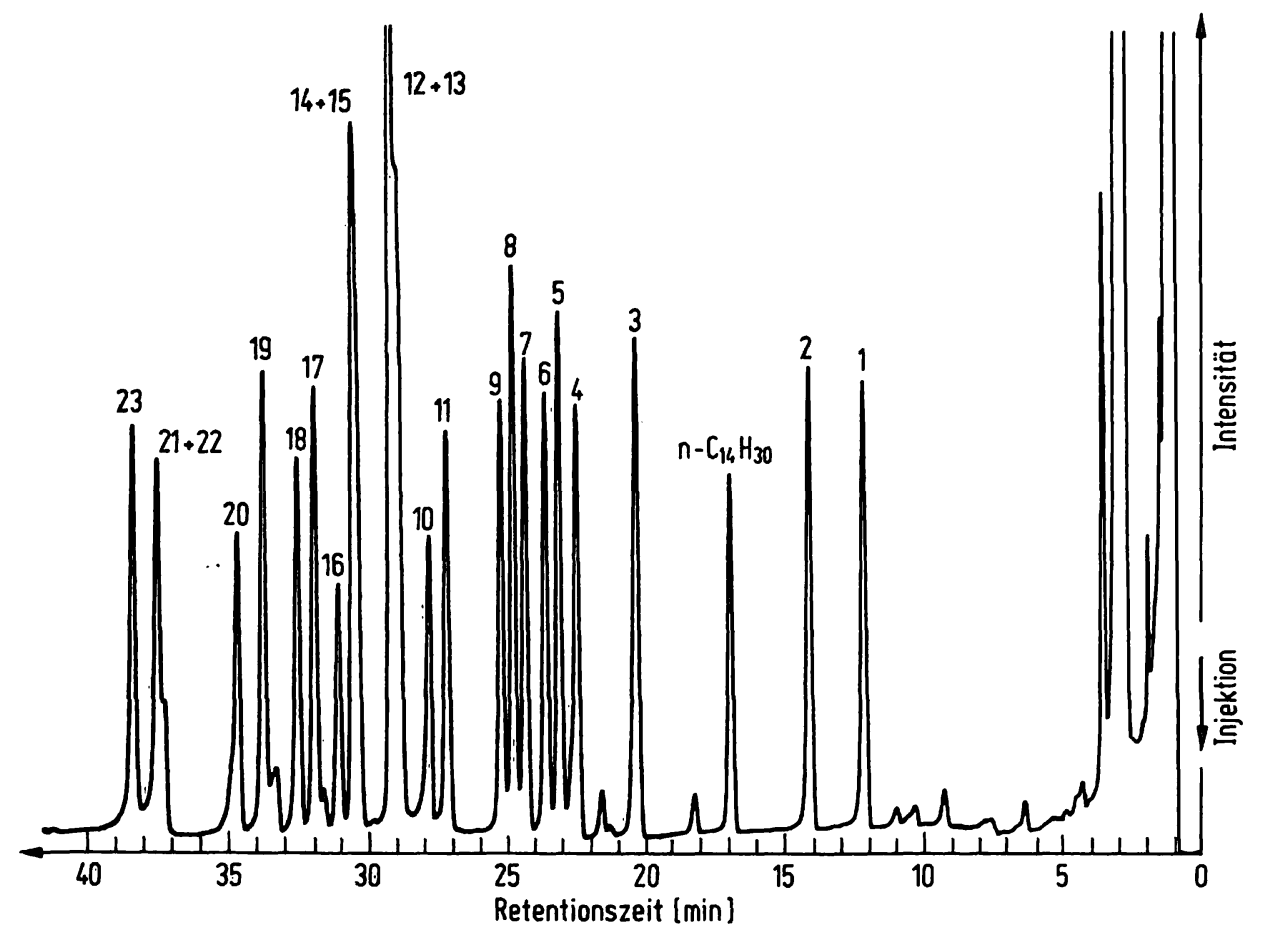

Abb. 1. Trennung silylierter aromatischer Carbonsäuren mit der gepackten Säule; chromatographische Bedingungen siehe Text; Verstärkung $8 \times 10^{2}$.

1 Benzoesäure; 2 Phenylessigsäure; 3 Mandelsäure; 4 Zimtsäure; 5 o-Hydroxyphenylessigsäure; 6 3-Phenylmilchsäure, $7 m$-Hydroxyphenylessigsäure; 8 -Hydroxybenzoesäure; $9 p$-Hydroxyphenylessigsäure, 10 Veratrumsäure; 11 Phenylbrenztraubensäure, 12 Vanillinsäure; 13 2,6-Dihydroxybenzoesäure; 140 -Hydroxyzimtsäure; 15 2,4-Dihydroxybenzoesäure; 16 Homogentisinsäure; 17 m-Hydroxyzimtsäure; 18 Vanillinmandelsäure; 19 p-Hydroxyzimtsäure; 20 Indol-3-essigsäure; 21 o-Hydroxyhippursäure, 22 Ferulasäure; 23 3,4-Dihydroxyzimtsäure.

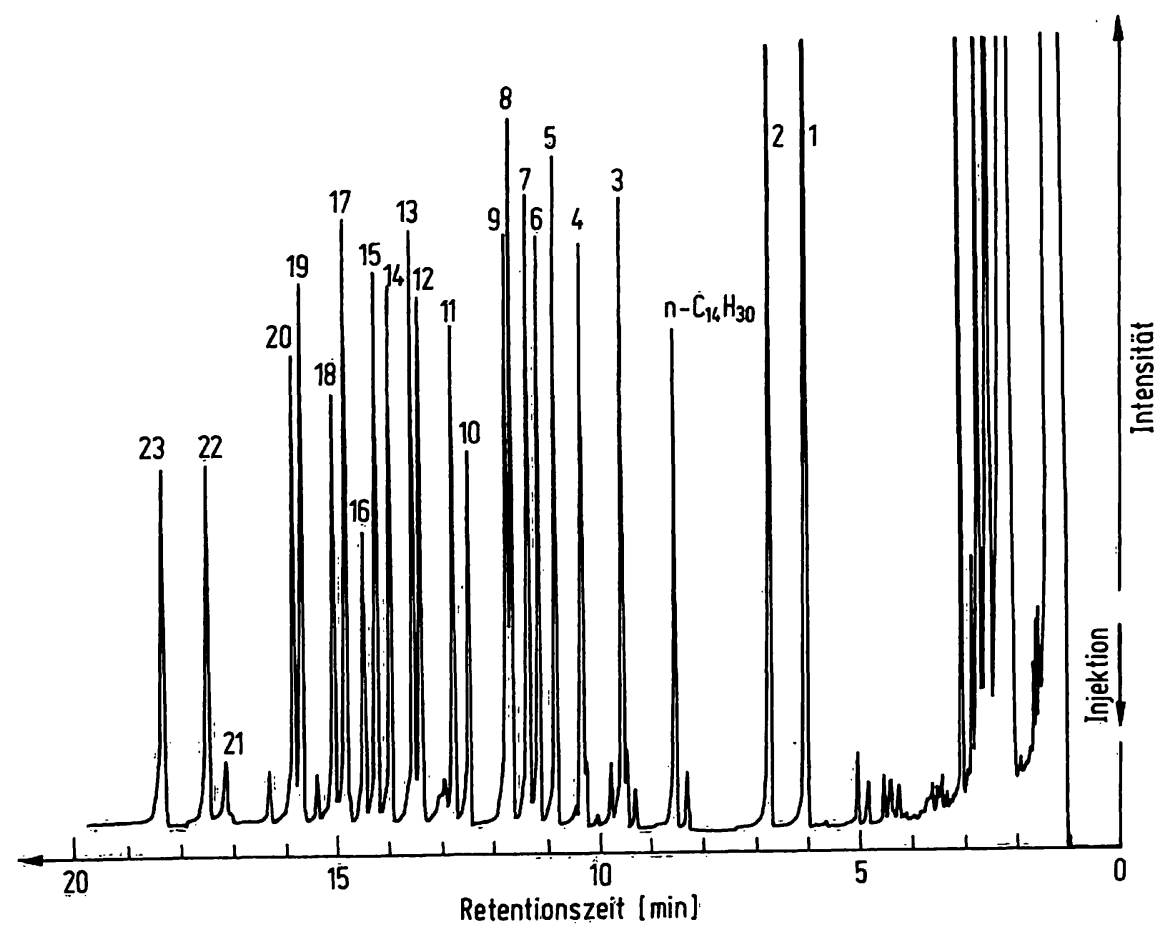

Abb. 2. Kapillarchromatogramm der gleichen Probe wie in Abb. 1; chromatographịsche Bedingungen siẹhe Text; Substanzbezeichnung wie Abb. 1, Verstärkung $1 \times 10^{2}$. 


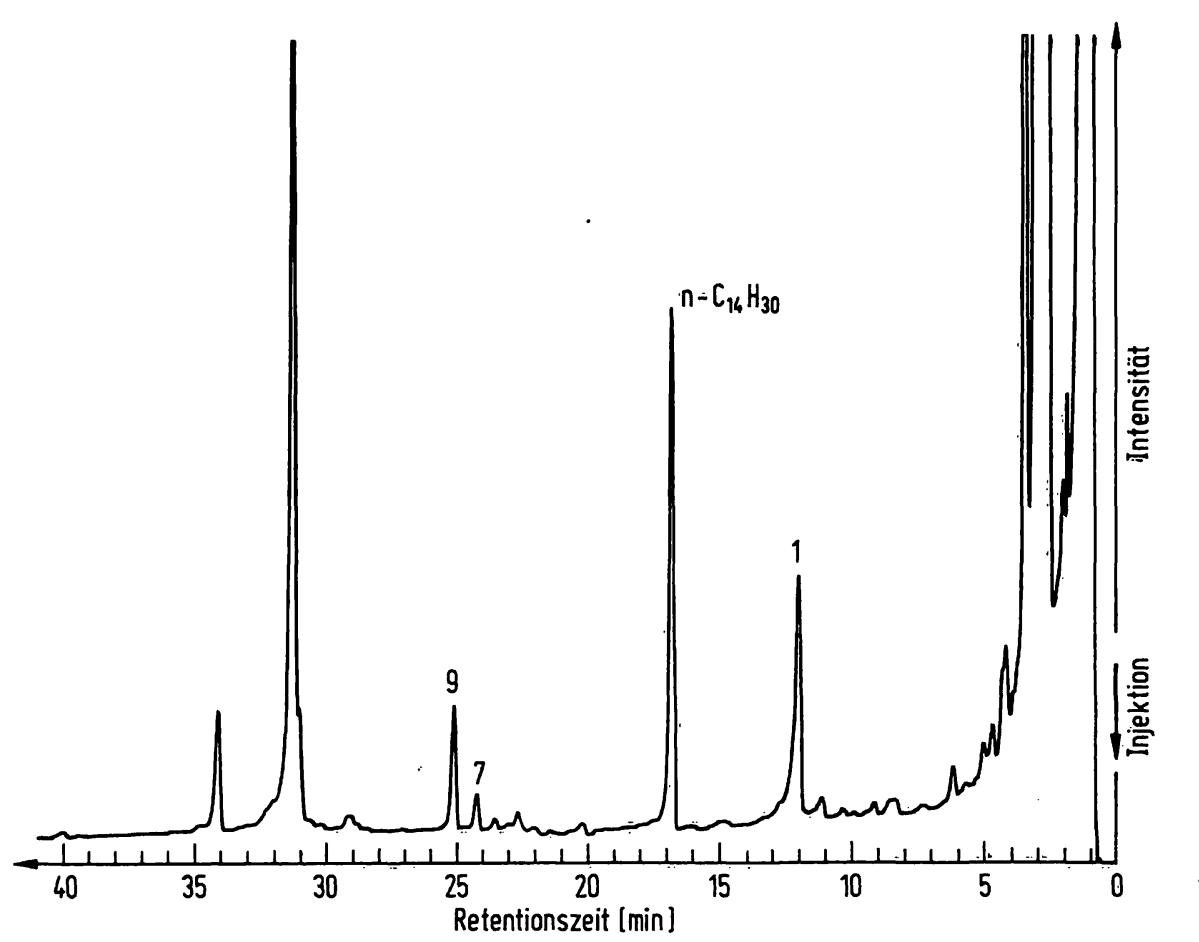

Abb. 3. Chromatogramm eines 24-h-Normalurins; gepackte Säule; chromatographische Bedingungen siehe Text; Substanz̈bezeichnung wie Abb. 1; Verstärkung $8 \times 10^{2}$.

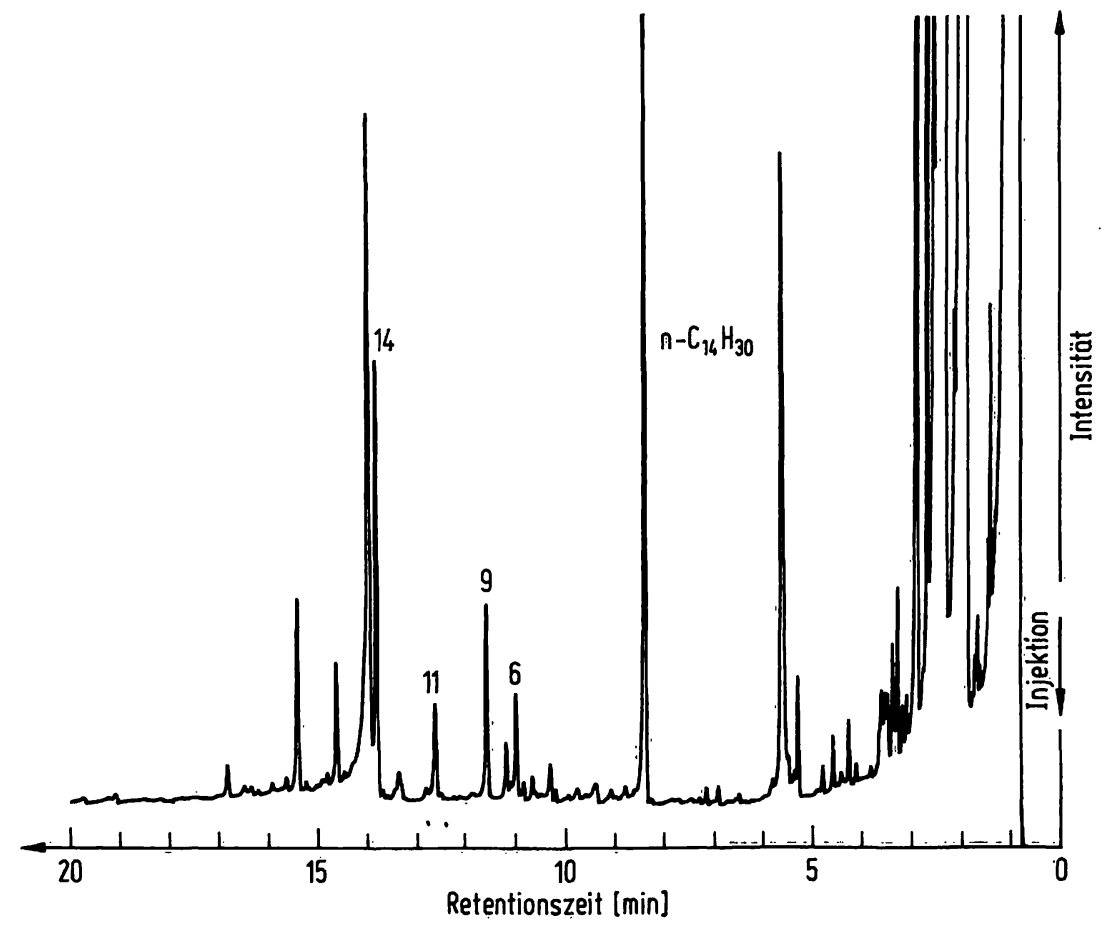

Abb. 4. Kapillarchromatogramm der gleichen Probe wie in Abb. 3; chromatograhpische Bedingungen siène Text; Substanzbezeichnung wie in Abb. 1; Verstärkung $1 \times 10^{2}$. Bemerkenswert ist der Peak 11 mit der Retentionșzeit vọn Phenylbrenżtraubensäure. Eine sichere Identifizierung war jedoch nicht möglich, da keine GC-MS-Koppélung zur Verfügung steht. 


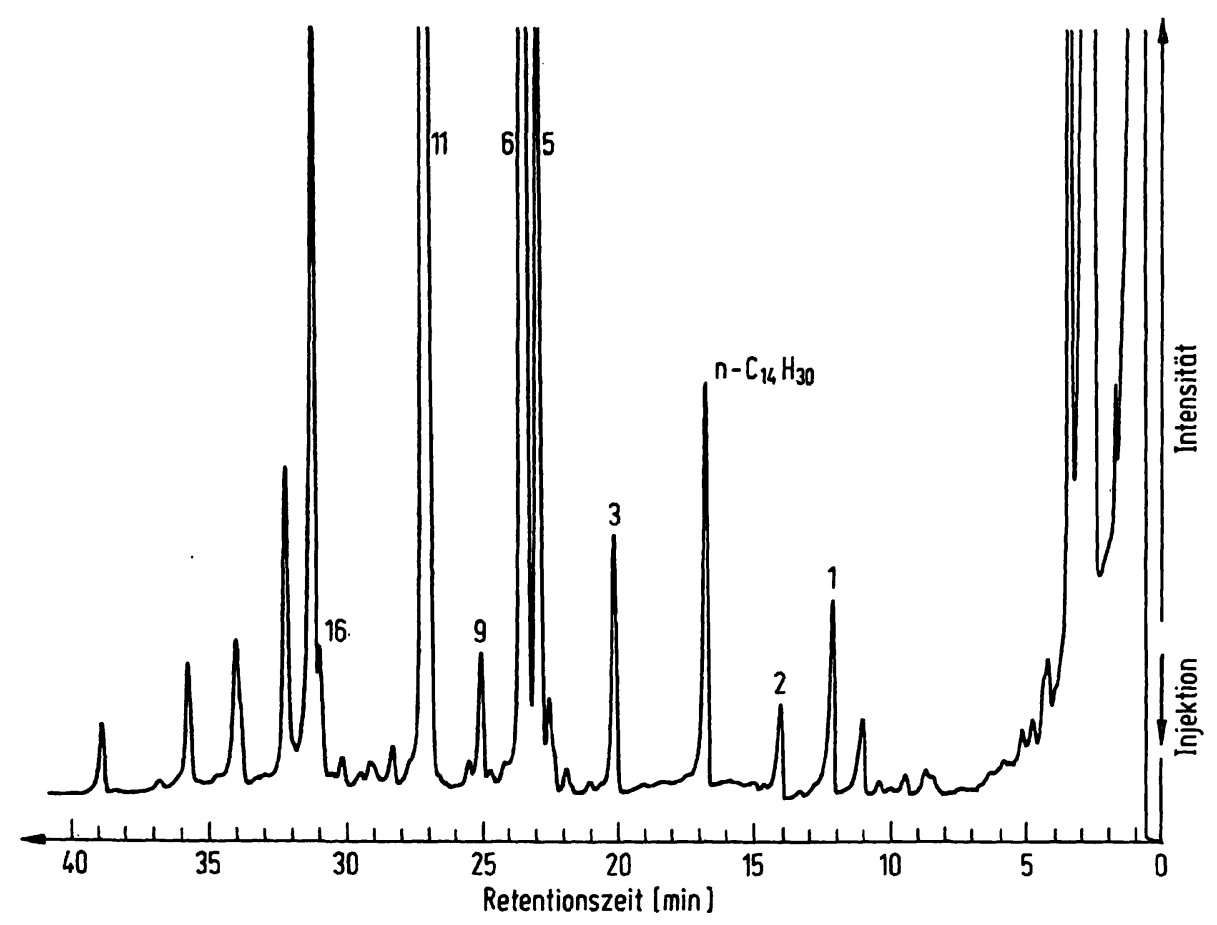

Abb. 5. Chromatogramm eines 24-h-Phenylketonurie-Urins; chromatographische Bedingungen siehe Text; Substanzbezeichnungen wie Abb. 1; Verstärkung $8 \times 10^{2}$.

Unter den gewählten Bedingungen werden Benzoesäure (Peak 1) und Harnstoff nicht getrennt. Bei Harnchromatogrammen ist dieser Sachverhalt meist an der unsymmetrischen Peakform ("heading") zu erkennen.

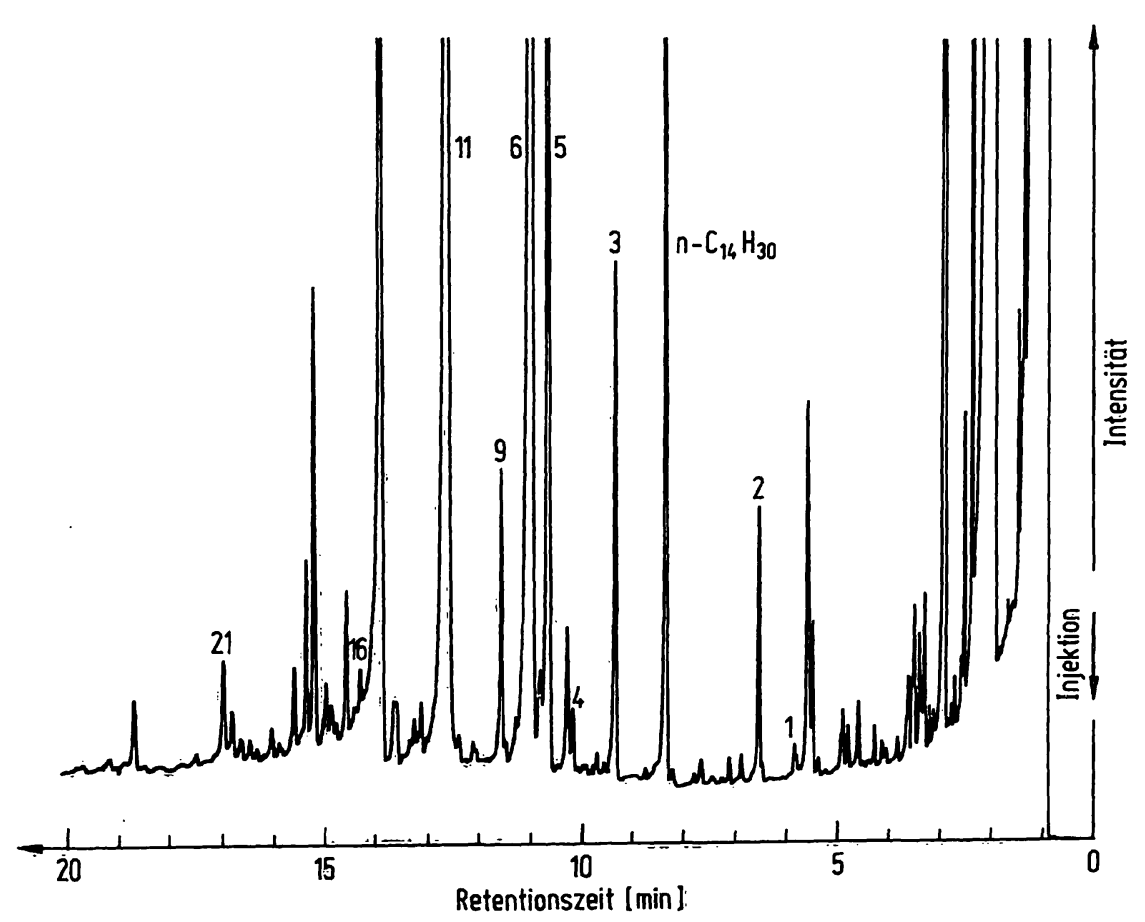

Abb. 6. Kapillarchromatogramm der gleichen Probe wie in Abb. 5; chromatographische Bedingungen siehe Text; Substanzbezeichnungen wie in $\mathrm{Abb}$. 1; Verstärkung $1 \times 10^{2}$.

Verglichen mit Abb. 5 wird für Benzoesäure (Peak 1) ein wesentlich kleinerer Peak gefunden, was auf die Trennschwierigkeit von Benzoesäure und Harnstoff mit der gepackten Säule zurückgeführt werden kann. 
Die quantitative Analyse zeigte, daß die Standardgeraden im betrachteten Konzentrationsbereich $(0,1 \mathrm{~g} / 1$ $2,5 \mathrm{~g} / \mathrm{l})$ linear verlaufen $(\mathrm{r} \approx 1)$. Tabelle 2 gibt die mit Hilfe einer linearen Regressionsanalyse ermittelten Werte des Korrelationskoeffizienten r, sowie Achsenabschnitt b und Steigung $m$ der Standardgeraden der einzelnen Substanzen wieder.

Tab. 2. Konstanten der Standardgerade $y=m x+b$ sowie Korrelationskoeffizient $r$ der einzelnen Substanzen. $(y=$ relative Impulszahl, $x=$ Konzentration in $g / 1 ; n=A n z a h l$ der Messungen).

\begin{tabular}{lllrl}
\hline Substanz & $\mathrm{r}$ & $\mathrm{m}$ & \multicolumn{1}{l}{$\mathrm{b}$} & $\mathrm{n}$ \\
\hline Benzoesäure & 0,998 & 0,937 & 0,000 & 34 \\
Phenylessigsäure & 0,997 & 0,833 & $-0,016$ & 34 \\
Mandelsäure & 0,999 & 1,039 & $-0,027$ & 34 \\
Zimtsäure & 0,995 & 0,991 & 0,002 & 34 \\
o-Hydroxyphenylessigsäure & 0,998 & 0,964 & $-0,020$ & 34 \\
3-Phenylmilchsäure & 0,995 & 0,905 & $-0,045$ & 34 \\
m-Hydroxyphenylessigsäure & 0,996 & 0,913 & $-0,045$ & 26 \\
p-Hydroxybenzoesäure & 0,997 & 1,121 & $-0,015$ & 34 \\
p-Hydroxy-phenylmilchsäure & 0,993 & 0,825 & $-0,045$ & 33 \\
Veratrumsäure & 0,972 & 0,589 & 0.026 & 33 \\
Phenylbrenztraubensäure & 0,988 & 0,886 & 0,062 & 21 \\
Vanillinsäure & 0,997 & 0,911 & $-0,026$ & 34 \\
2,6-Dihydroxybenzoesäure & 0,994 & 0,987 & $-0,014$ & 34 \\
o-Cumarsäure & 0,997 & 0,936 & $-0,103$ & 34 \\
o,p-Hydroxybenzoesäure & 0,987 & 0,975 & 0,008 & 33 \\
Homogentisinsäure & 0,956 & 0,884 & 0,012 & 20 \\
m-Cumarsäure & 0,991 & 1.105 & -0.013 & 34 \\
Vanillinmandelsäure & 0,984 & 0,882 & $-0,078$ & 27 \\
$p$-Cumarsäure & 0,996 & 1,012 & $-0,080$ & 34 \\
Ferulasäure & 0,995 & 0,739 & $-0,093$ & 31 \\
m, p-Dihydroxyzimtsäure & 0,988 & 0,881 & $-0,072$ & 31 \\
& & & &
\end{tabular}

Die Anwendbarkeit der beschriebenen Methode möge durch die quantitative Analyse der Phenylalaninmetaboliten im Urin von vier erwachsenen Phenylketonurie-Patienten demonstriert werden (Tab. 3).

\section{Literatur}

1. Gottschalk, G. (1966), Einführung in die Grundlagen der chemischen Materialprüfung, S. Hirzel-Verlag, Stuttgart.

2. Weber, E. (1967), Grundriß der biologischen Statistik; Gustav Fischer Verlag, Stuttgart.
Tab. 3. Konzentration aromatischer Carbonsäuren im 24-h-Urin erwachsener Phenylketonurie-Patienten; Konzentration in $\mathrm{mg} / \mathrm{g}$ Kreatinin.

\begin{tabular}{|c|c|c|c|c|}
\hline & \multicolumn{4}{|c|}{ Patienten } \\
\hline & H. Fi & H. S. & H. Fö. & S. h. \\
\hline Phenylessigsäure & 22,83 & 25,81 & 25,01 & 28,07 \\
\hline Mandelsäure & 25,92 & 25,59 & 34,45 & 27,10 \\
\hline $\begin{array}{l}\text { o-Hydroxyphenyl- } \\
\text { essigsäure }\end{array}$ & 71,76 & 59,84 & 89,86 & 68,79 \\
\hline 3-Phenylmilchsäure & 317,73 & 169,67 & 168,56 & 360,16 \\
\hline $\begin{array}{l}p \text {-Hydroxyphenyl- } \\
\text { essigsäure }\end{array}$ & 34,28 & 47,98 & 31,24 & 30,08 \\
\hline $\begin{array}{l}\text { Phenylbrenz- } \\
\text { traubensäure }\end{array}$ & 654,48 & 420,48 & 507,15 & 313,67 \\
\hline $\begin{array}{l}\text { Phenylalanin } \\
\text { im Serum (mg/l) }\end{array}$ & 163 & 239 & 218 & 199 \\
\hline
\end{tabular}

Die im Urin dieser Homozygoten gefundenen Konzentrationen an 3-Phenylmilchsäure und Phenylbrenztraubensäure sind 2-3 mal so hoch, wie die höchste für die Standardkurve verwandte Konżentration. Ein separat durchgeführter Versuch zeigte, daß die Standardgeraden der Tabelle 2 auch in diesem Konzentrationsbereich gültig sind. Wie aus Tabelle 3 weiterhin zu entnehmen, liegen die gefundenen Konzentrationen an $o$-Hydroxyphenylessigsäure, 3-Phenylmilchsäure und Phenylbrenztraubensäure um 50-60\% unter den Literaturwerten eines entsprechenden Patientenkollektivs (3). Dies ist wahrscheinlich auf die niedrigeren Phenylalaninwerte im Serum unserer Patienten zurückzuführen.

\section{Danksagung}

Wir danken Frau D. Schmitt für die wertvolle technische Hilfe bei der Durchführung dieser Arbeit.

3. Chalmers, R. A. \& Watts, R. W. E. (1974), Clin. Chim. Acta $55,281-000$.
Dr. P. Wardenbach Institut für Humangenetik Wilhelmstraße 31 5300 Bonn 